\title{
Análisis de las contribuciones del aprendizaje-servicio al estudiantado de una universidad sudafricana desde el enfoque de las capacidades
}

\author{
Analysis from the Capabilities Approach of the Contributions of Service Learning for \\ Students of a South African University
}

\section{Análise das contribuições da aprendizagem serviço para estudantes de uma universidade sul-africana a partir de uma abordagem de competências}

Carmen Monge-Hernández

Universidad Nacional

ROR:: https://ror.org/01t466c14

Heredia, Costa Rica cmonge@una.cr

D https://orcid.org/0000-0001-7435-8628

Alejandra Boni-Aristizábal Universitat Politècnica de València'

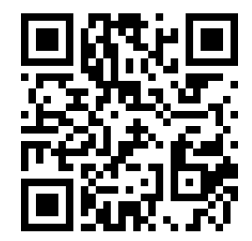
Valencia, España aboni@dpi.upv.es https://orcid.org/0000-0003-3945-4336

Merridy Wilson-Strydom University of the Free State Centre for Research on Higher Education and Development Free State, South Africa merridywilsonstrydom@gmail.com https://orcid.org/0000-0002-6591-0473

Recibido • Received • Recebido: 03 / 09 / 2018

Corregido • Revised • Revisado: 07 / 02 / 2020

Aceptado • Accepted • Aprovado: 07 / 07 / 2020

\footnotetext{
${ }^{1}$ Ingenio (CSIC- Universitat Politècnica de València).
} 
http://doi.org/10.15359/ree.24-3.3

http://www.una.ac.cr/educare

educare@una.cr

Resumen: Este artículo aborda la contradicción entre la formación orientada a las capacidades técnicas y la centrada en capacidades socialmente valoradas. Su propósito es valorar experiencias pedagógicas que contribuyen a la ampliación de capacidades profesionales valiosas para el estudiantado y la sociedad. A partir del enfoque de capacidades para el desarrollo humano (ECDH), se analiza la contribución a la ampliación de las capacidades del estudiantado que promueven las prácticas curriculares de aprendizaje-servicio (ApS), impulsadas por la Universidad del Estado Libre, Sudáfrica. El análisis se sustenta en la aplicación de diferentes técnicas de corte cualitativo: revisión documental, observación participante y entrevistas semiestructuradas. Los resultados visibilizan la expansión de capacidades socialmente valiosas en el estudiantado y orientan recomendaciones de cambios institucionales para la integración curricular y la valorización institucional del ApS, como una experiencia formativa transformadora.

Palabras claves: Educación superior; desarrollo humano; enfoque de las capacidades; aprendizajeservicio.

Abstract: This article addresses the contradiction between training oriented to technical capabilities and training focusing on socially valued capabilities. Its purpose is to value pedagogical experiences that contribute to the expansion of valuable professional skills for students and society. Based on the capabilities approach for human development (CAHD), we analyzed the contribution to the expansion of student capacities that is promoted by curricular service-learning (ApS) practices, developed at the University of the Free State (UFS), South Africa. The analysis is based on the application of the following qualitative techniques: documentary review, participant observation, and semi-structured interviews. The results make visible the expansion of socially valued capacities in the students and serve as a guide for recommendations of institutional changes for curricular integration and institutional valorization of $\mathrm{ApS}$, as a transforming formative experience.

Keywords: Higher education; human development; capabilities approach; service learning.

Resumo: Este artigo aborda a contradição entre formação orientada às capacidades técnicas e as focadas em capacidades valorizadas socialmente. Seu objetivo é valorizar experiências pedagógicas que contribuam à expansão de capacidades profissionais que são valorizadas pelos estudantes e pela sociedade. Com base na abordagem do Enfoque de Capacidades para o Desenvolvimento Humano $(E C D H)$, analisa-se a contribuição para a expansão das capacidades de estudantes que promovem às práticas curriculares de aprendizagem em Serviço (AS), promovidas pela Universidade do Estado Livre da África do Sul. A análise baseia-se na aplicação de diferentes técnicas de corte qualitativo: revisão documental, observação participante e entrevistas semiestruturadas. Os resultados tornam visível a expansão das capacidades socialmente valiosas no estudo e orientam recomendações para mudanças institucionais para a integração curricular e a valorização institucional do AS, como experiência formativa transformadora.

Palavras-chave: Educação superior; desenvolvimento humano; enfoque das capacidades; aprendizagem-serviço. 


\section{Introducción}

En los últimos años, se ha incrementado el debate en torno al rol de las universidades en el presente siglo y sobre cómo pueden formar profesionales capaces de aportar a la lucha contra la pobreza, la desigualdad y la degradación ambiental (Santos, 2007; Walker y McLean, 2013). Una de las mayores preocupaciones es la influencia que ejerce el modelo de pensamiento económico dominante en la política de educación superior en el ámbito mundial. Esto se refleja en las perspectivas hegemónicas que influencian y presionan a las universidades para centrar sus objetivos en criterios gerenciales internacionales, orientados por la lógica del capital humano (Walker, 2012), y que hacen que la educación superior se concentre en conseguir logros que permitan el incremento de la productividad y el desarrollo económico (Alarcón y Guirao, 2013), escalar en los rankings globales (Wilson-Strydom y Walker, 2015) y formar en función de la empleabilidad (Suleman, 2016).

Sin embargo, a nivel internacional se destaca un consenso respecto al compromiso ineludible de la universidad pública con la sociedad. La UNESCO (1998), en el Artículo 6 de la Declaración Mundial sobre la Educación Superior en el Siglo XXI, insta a las universidades a que alineen su función al servicio de la sociedad, por medio de acciones inter y transdiciplinarias, con la finalidad de reducir los problemas sociales que viven nuestras comunidades, como son la enfermedad, la pobreza, la violencia, el deterioro ambiental y el analfabetismo. De esta misma perspectiva, hay importantes planteamientos que adhieren el enfoque de las capacidades para el desarrollo humano a la educación superior, tal es el caso de Walker y McLean (2013), quienes enfatizan en la necesidad de un modelo de universidad que forme integralmente y disponga a la sociedad de profesionales universitarios y universitarias con conocimientos, habilidades y valores que les permitan asumir activa y críticamente los retos de desarrollo y combatir las desigualdades sociales.

Actualmente, un gran número de universidades públicas de diversas partes del mundo realizan esfuerzos importantes orientados hacia esa formación integral y transformación social necesaria del estudiantado; algunas de ellas han apostado a conseguirlo por medio de la interacción pedagógica con la comunidad (Erasmus, 2007). Tal es el caso de la Universidad del Estado Libre de Sudáfrica (en adelante UFS, por su nombre en inglés University of the Free State) que aprobó, en el 2006, la política de community engagement, que contiene una categoría que incluye el ApS (Service Learning en inglés) en el plan de estudios, con el fin de lograr la reflexión y la transformación social necesarias para superar las condiciones de pobreza y su difícil pasado sociopolítico.

Considerando la necesidad de evidenciar oportunidades y logros individuales estudiantiles generados con el ApS, se utiliza como soporte teórico el ECDH (Nussbaum, 2001; Sen, 2000). Este estudio se propone avanzar en la comprensión de la ampliación de las capacidades profesionales valiosas para la sociedad que favorece un proceso formativo que incorpora las prácticas de ApS de interrelación con la comunidad. El análisis busca también aportar recomendaciones para 
disponer de una institucionalidad universitaria que potencie el desarrollo de la integralidad en las capacidades y logros en el estudiantado. El ECDH, un enfoque pluridimensional del desarrollo (Alkire, 2002), ofrece un marco teórico que facilita el análisis sustentado en valores como bienestar, equidad y diversidad, empoderamiento y participación, y sostenibilidad (Boni y Gasper, 2011), desde una visión de justicia y prosperidad social. Sus postulados nos sirven de sustento al ideario de profesionalismo al que aspiramos en nuestras universidades públicas y que demanda la sociedad actual (Walker y McLean, 2013), más inclusiva, social, responsable y participante de las problemáticas sociales globales (Boni y Gasper, 2011).

Para mostrar los resultados de este estudio se presenta, inicialmente, una breve descripción de los principales argumentos teóricos propuestos por el ECDH en la educación superior, con énfasis en el índice de capacidades de las personas profesionales de Walker y McLean (2013); seguidamente, se contextualiza el estudio de caso. Luego, se describe la metodología utilizada y se exponen los principales resultados obtenidos, consistentes en una descripción de la ampliación de las capacidades logradas por el estudiantado a partir de las prácticas de ApS, así como los factores de conversión intervinientes. Se cierra con una discusión donde se reflexiona sobre los resultados del ApS e inevitables implicaciones estratégicas para la UFS, orientadas al fortalecimiento y la sostenibilidad del ApS, en pos de la continuidad y profundización de capacidades del estudiantado para un profesionalismo centrado en el desarrollo humano.

\section{EI ECDH en la educación superior}

El ECDH es un marco conceptual normativo para la evaluación y el diseño del estado de bienestar individual y de las políticas de bienestar de las instituciones (Robeyns, 2017), como es el caso de las universidades. Dentro de sus aportaciones, está la posibilidad de centrar la atención en los valores públicos, como derechos humanos, libertad y justicia social; razón por la cual es considerado un paradigma alternativo. Este enfoque rechaza los discursos dominantes sobre la sociedad, la pobreza y el desarrollo. Propone indagar en todo aquello que permite que las personas vivan mejor y logren mayor calidad de vida (Sen, 2000).

Para este enfoque, la sociedad en su conjunto y las organizaciones públicas, entre ellas las universidades, tienen la responsabilidad de contribuir a que la totalidad de miembros de la sociedad tenga oportunidades para incrementar las capacidades humanas centrales (Nussbaum, 2001). Las capacidades humanas centrales de Nussbaum (2001) comprenden una serie de capacidades interrelacionadas entre sí, que van desde la libertad de vivir; tener una buena salud e integridad corporal; utilizar los sentidos, la imaginación, el pensamiento, las emociones y la razón práctica; vivir con los otras personas y para ellas; hasta la de jugar y participar, entre otras. Esas capacidades son las que les permiten, a las personas, tener una vida digna y, por tanto, las orientadoras de las políticas y acciones de desarrollo. 
Tanto Nassubaun (2001) como Sen (2000) consideran la educación como un medio para ampliar las oportunidades y los logros plurales de las personas. De ahí la consideración sobre la gran valía de las acciones pedagógicas y políticas encaminadas a generar cambios en las universidades. En este enfoque, la ampliación de oportunidades o libertades es fundamental para encaminar a las personas al florecimiento humano (Wilson-Strydom y Walker, 2015). Esto apunta al fomento de entornos educativos potencializadores de ese florecimiento; a proponerse formar a las personas para que florezcan y logren entrelazar la capacidad de agencia con la moralidad, capacidad de agencia entendida como "persona que actúa y provoca cambios y cuyos logros pueden juzgarse en función de sus propios valores y objetivos" (Sen, 2000, p. 35). Este vínculo agencia - moralidad le implica, a la persona, tomar decisiones autónomas y actuar en consecuencia, como una persona que actúa, vive y razona de una forma moral, al incorporar los valores. Esto le significaría al estudiantado o profesionales del futuro, tener la sensibilidad y la comprensión motivacional para ser un buen ser humano y, por justicia social, sostener la lucha constante y el compromiso para propiciar mejoras en el bienestar de otros seres, dentro y fuera de la universidad (Wilson-Strydom y Walker, 2015).

Como primera idea central de este tipo de análisis, el enfoque propone estudiar las capacidades y funcionamientos de la persona, en términos de capacidades reales (libertades) y funcionamientos (logros) que tiene la persona para ser y hacer lo que valora (Sen, 2000). Por tanto, las capacidades corresponden al set de combinaciones de funcionamientos que pueden conseguir las personas en áreas que valoran importantes para su vida (Sen, 2000). Esto llama a razonar entorno a aquello que las personas (el estudiantado) valoran ser y hacer en su vida y promover, por medio del currículo y los arreglos pedagógicos, aumentos de sus libertades (o capacidades) para alcanzar sus aspiraciones y para que logren avances en términos de lo que consideran bueno para sus vidas (Wilson-Strydom y Walker, 2015).

La segunda idea central es la agencia, antes expuesta, y la tercera lo constituyen los factores de conversión, que son factores que determinan el grado en que una persona puede transformar un recurso en un funcionamiento (Robeyns, 2017). La teoría sugiere la comprensión de los factores personales (habilidades, condición psicológica), sociales (marco político, normas, relaciones de poder) y, por último, los factores ambientales (medios de transporte, ubicación geográfica) (Robeyns, 2017; Sen, 2000). Esto conllevaría a indagar sobre los factores que influyen en cómo el estudiantado convierte las oportunidades (o libertades) derivadas del ApS en funcionamientos reales o logros que valora. Por tanto, el ECDH es el lente utilizado para entender lo que promueve el ApS en el estudiantado y las condiciones de la UFS que posibilitan u obstaculizan esos resultados en este grupo.

Desde esta perspectiva del ECDH, Walker y McLean (2013) han ampliado las comprensiones normativas sobre cómo debería ser una educación universitaria encaminada al bien público y hacia un profesionalismo con capacidades profesionales para desarrollarse a favor de la justicia 
social de los grupos y sectores más vulnerables, por medio del índice de capacidades estudiantiles elaborado a partir de información empírica extraída de estudiantes y documentación de varias universidades sudafricanas. Su estudio esclarecela manera en que la universidad puede propiciar una transformación profesional que no sólo genere capacidades para ocupar un puesto laboral, sino que favorezca la expansión de capacidades y funcionamientos en áreas vitales para el desarrollo humano. Este índice identifica las capacidades a las que las universidades deben orientar sus objetivos, para conseguir una formación que genere transformaciones individuales y colectivas, dentro y fuera del ejercicio de la profesión; por ejemplo, en la lucha contra las condiciones de pobreza e inequidad que les rodea (Figura 1).

Figura 1: Capacidades profesionales deseadas, meta para la educación universitaria sudafricana

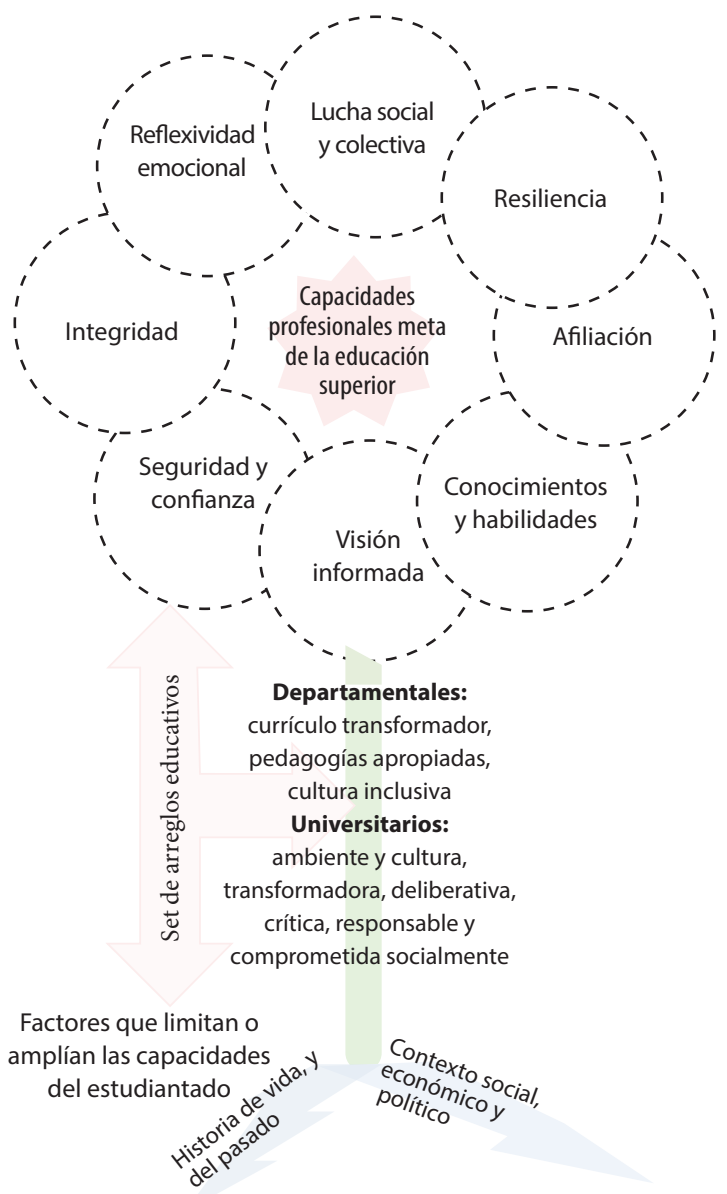

Nota: Elaboración propia a partir de Walker y McLean (2013). 
Walker y McLean (2013) conceptualizan la visión informada como aquella capacidad que permite al estudiantado lograr la comprensión de la influencia que ejerce el contexto histórico, socioeconómico y las estructuras culturales y políticas en la vida de las personas y el ejercicio de su profesión. Los conocimientos y habilidades les permiten la aplicación de conocimientos especializados en el campo profesional, aplicación basada en dicha visión informada; comprenden las habilidades humanas, cognitivas, intelectuales y gestión de proyectos, entre otras. Por su parte, la capacidad de afiliación se logra a partir del acierto del trabajo social para la construcción de relaciones con las personas de distintos perfiles, incluye esfuerzos hacia la igualdad de respeto, transformación social y empoderamiento de las personas.

La lucha colectiva y social es la capacidad estudiantil que permitiría orientar el ejercicio de la profesión hacia el liderazgo, el empoderamiento social y la promoción de los derechos humanos. La resiliencia favorece que se enfrente el sufrimiento del pasado, supere los obstáculos cotidianos y genere ideales, motivación y persistencia para alcanzar logros en el tiempo. La reflexividad emocional se deriva de la transformación personal, potenciará sus motivaciones para impulsar la transformación social. La integridad les motiva a sostener un comportamiento ético y honesto y a tener la valentía para defender lo que es correcto. Finalmente, la seguridad y confianza les permite acercarse a su rol profesional, lo que posibilita la expansión de su agencia y poder para contribuir al cambio social deseado.

\section{Estudio de caso en la Universidad Estado Libre de Sudáfrica}

Nos adentramos en la UFS, una de las universidades públicas más antiguas de Sudáfrica, fundada en 1904. Su campus principal está en la ciudad de Bloemfontein, la capital judicial de Sudáfrica, que pertenece a la provincia de Free State. Esta universidad tiene cerca de 4200 empleados y empleadas, y atiende alrededor de 34000 estudiantes (UFS, 2015).

La incorporación del community engagement en Sudáfrica y, por tanto, en la UFS, se afianzó con las acciones de la nueva democracia y la agenda de reconstrucción post apartheid (Hall, 2010; Preece et al., 2012). Según Erasmus y Albertyn (2014), el concepto de community engagement, cuya traducción al castellano es compromiso con la comunidad, está asociado internacionalmente a términos como acción social, extensión, compromiso cívico o interacción con la comunidad. A partir de 1997, las disposiciones nacionales impulsadas promovieron la inserción del community engagement, aceptándolo como uno de los tres componentes fundamentales de la educación superior (Figura 2). Se concibe como un componente integral de la docencia y la investigación, debido al enriquecimiento del proceso de enseñanza y aprendizaje que se logra con la comprensión e interiorización profunda del contexto, la localidad y la aplicación (White Paper 1997, citado por Lazarus et al., 2008). 
En el 2006, la UFS aprobó la política 06.1 de servicio comunitario, que contiene el sustento conceptual-metodológico y de estrategia operativa del community engagement. Esta política se propone lograr la transformación social y construir e intercambiar los conocimientos, habilidades, experiencia y recursos necesarios para desarrollar y sostener la sociedad (UFS, 2006). Para esto, dispone de tres servicios según la Figura 2.

Figura 2: Categorías de community engagement de la UFS

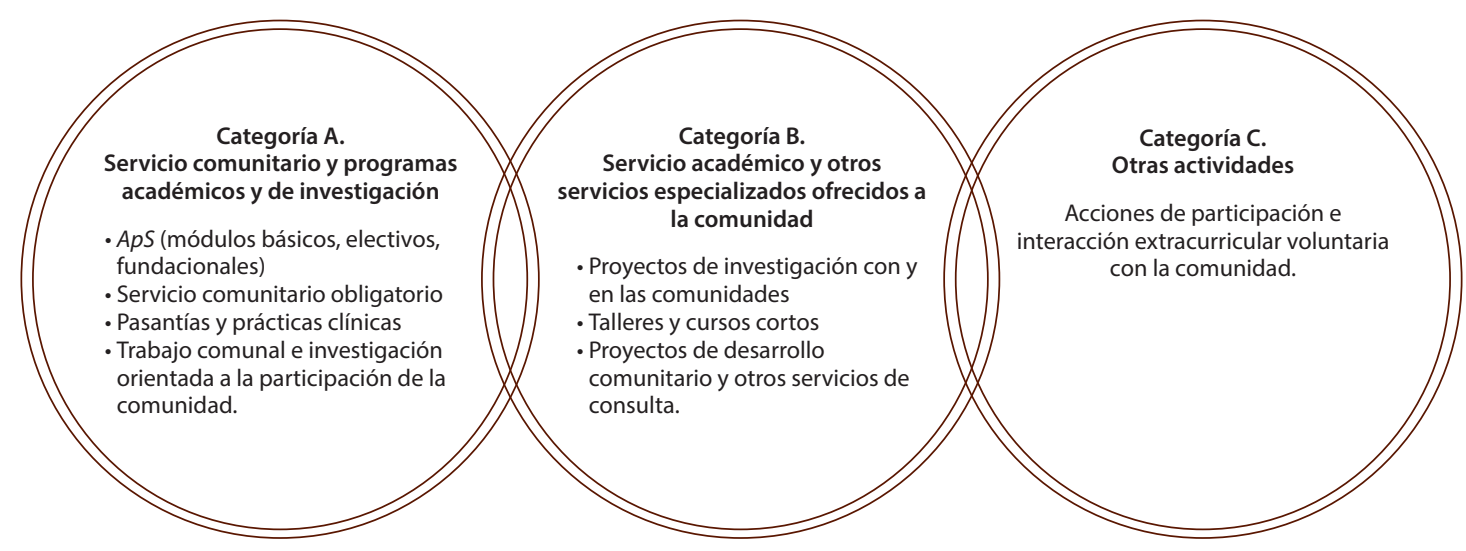

Nota: Elaboración propia a partir de UFS (2006).

En la categoría $A$, el ApS es un enfoque educativo, que puede adecuarse a la dinámica de cada carrera, para el desarrollo de experiencias de aprendizaje dentro del currículo, con el fin de que el estudiantado tenga la posibilidad, por un lado, de participar activamente en el abordaje de las demandas comunales, debidamente contextualizadas y planificadas y, por otro, reflexionar profundamente sobre la vinculación que tiene el contenido curricular y la dinámica de la comunidad (UFS, 2006).

Previo a esta política, la UFS tenía experiencia desarrollada a inicios de la década de los 90, con ocho asignaturas de ApS en las Facultades de Ciencias de la Salud y de Ciencias Sociales (Fourie, 2003). Si bien hoy no todas las carreras han logrado aún incorporar el ApS, pues es una opción voluntaria, las estadísticas son bastante satisfactorias. Según los informes del periodo 2011-2014, la UFS en promedio anual logra: 60 módulos (asignaturas), participación de 77 docentes y de 3200 estudiantes y 80000 horas de trabajo pedagógico en las comunidades y con ellas (UFS, 2011; 2012a; 2013; 2014). 


\section{Metodología}

En este estudio, se usa el enfoque cualitativo como medio para recoger y analizar los datos. Los datos cualitativos son útiles para los estudios que tratan de comprender las opiniones y percepciones que tienen significancia para las personas en un área específica (Corbetta, 2007; Valles, 1999). Se razona tomar de estudio de caso las experiencias de ApS, que UFS realiza desde la década de los 90 (Fourie, 2003), por sus objetivos de formación integral y de transformación estudiantil. El ApS constituye un espacio pedagógico formal propicio para estudiar las prácticas de interacción con la comunidad como medio para avanzar en la creación de capacidades profesionales propuestas por Walker y McLean (2013).

Paraconseguir el objetivo dela investigación,seaplicaron trestécnicas:revisión documental, entrevistas semiestructuradas y observación participante (Tabla 1), para dar respuesta a las dos preguntas orientadoras del estudio: ¿A cuáles de las capacidades identificadas por Walker y McLean (2013) contribuye el ApS en el estudiantado participante? ¿Cuáles son algunos de los factores (institucionales, sociales o personales) que favorecen o restringen el desarrollo del ApS y su contribución al desarrollo humano del estudiantado?'. Tratamos de entender, por un lado, el contexto y las aportaciones más valoradas que genera el ApS en el estudiantado (capacidades y funcionamientos), basándonos en las capacidades identificadas por Walker y McLean (2013) $y$, por otro, las condiciones necesarias para garantizar las capacidades estudiantiles (factores de conversión), derivadas del ejercicio pedagógico de ApS.

Tabla 1: Compendio de técnicas utilizadas

\begin{tabular}{|c|c|c|}
\hline Técnica & Objetivo & Fuentes \\
\hline Revisión documental & $\begin{array}{l}\text { Obtener la perspectiva institucional y documental de las } \\
\text { experiencias de ApS que permita la contextualización del } \\
\text { estudio de caso y la aproximación a la realidad social a estudiar }\end{array}$ & $\begin{array}{l}\text { - Planes de estudio. } \\
\text { - Normativa institucional. } \\
\text { - Informes de ApS }\end{array}$ \\
\hline $\begin{array}{l}\text { Entrevistas } \\
\text { semiestructuradas } \\
\text { individuales }\end{array}$ & $\begin{array}{l}\text { Recuperar las narrativas de los sujetos y testimonios cortos } \\
\text { de otros sujetos vinculados con los procesos de ApS, que } \\
\text { cuentan sobre las capacidades, funcionamientos y factores de } \\
\text { conversión en dichos procesos }\end{array}$ & $\begin{array}{l}\text { - Grabaciones con los } \\
\text { relatos de las personas. } \\
\text { - Transcripciones completas } \\
\text { de las entrevistas }\end{array}$ \\
\hline $\begin{array}{l}\text { Observación } \\
\text { participante }\end{array}$ & $\begin{array}{l}\text { Comprender las experiencias, las relaciones y significancias } \\
\text { de los procesos para las personas participantes }\end{array}$ & $\begin{array}{l}\text { - Anotaciones. } \\
\text { - Fotografías. } \\
\text { - Grabaciones }\end{array}$ \\
\hline
\end{tabular}

Nota: Elaboración propia.

\footnotetext{
${ }^{1}$ Este estudio se desarrolló como parte de las prácticas externas internacionales del master de Cooperación al
} Desarrollo de la UPV, a partir del trabajo final de graduación (Monge, 2016). 
Buscamos visibilizar el potencial de contribución del ApS en el estudiantado, desde el ECDH, y la red multidimensional de factores influyentes que disponen y necesitan los individuos (el estudiantado), para que logren o puedan alcanzar ciertos logros satisfactorios para la sociedad o un cierto bienestar individual y, posteriormente, usar esa información para recomendar intervenciones (políticas, cambio institucional, asignación de recursos, etc.) que garanticen la amplificación de la gama de capacidades y funcionamientos promovidos en las personas participantes (Robeyns, 2017). Para esto, se realiza una revisión documental, de los planes estratégicos, la política de servicio comunitario, informes y material publicado.

Las entrevistas se realizaron a diferentes participantes del ApS en UFS (estudiantes, profesorado o equipo docente y de gestión académica y organizaciones comunitarias), según la Tabla 2.

Tabla 2: Entrevistas semiestructuradas aplicadas

\begin{tabular}{lcc}
\hline \multicolumn{1}{c}{ Criterio de selección de personas entrevistadas } & Código Cantidad \\
\hline Alto nivel de decisión en la UFS, labora en gestión académica & Gestora & 1 \\
$\begin{array}{l}\text { Experiencia y responsabilidad de ejecución de la política CE/ApS, labora en gestión } \\
\text { académica }\end{array}$ & Gestora \\
$\begin{array}{l}\text { Experiencia y docencia actual; alto liderazgo y compromiso con el ApS } \\
\text { Estudiante de ApS en el momento de la entrevista }\end{array}$ & Profesora & 3 \\
$\begin{array}{l}\text { Trabajador/a de una organización no gubernamental (ONG) con experiencia de alianzas } \\
\text { con la UFS, participante de ApS }\end{array}$ & 3 & 2 \\
\hline
\end{tabular}

Nota: Elaboración propia.

La caracterización básica de las actividades de ApS en observación participante está relacionada con las experiencias y fueron elegidas al azar, considerando las posibilidades durante el tiempo presente en terreno y la aceptación por parte de los equipos de UFS. Los criterios para la selección de la muestra fueron: i) acciones estudiantiles desarrolladas desde el plan de estudios universitarios, ii) participación activa del estudiantado en el trabajo con la comunidad mediante el desarrollo práctico de conocimientos (la praxis) y el diálogo con las personas externas, iii) orientación de las acciones hacia la contribución significativa a personas o grupos sociales en condición de pobreza y desigualdad y iv) aceptación voluntaria de participación en la investigación, del equipo del Departamento de Community Engagement, así como del equipo docente y del estudiantado de UFS.

De los 60 módulos de ApS en ejecución durante el 2015 en siete facultades (UFS, 2015), se utilizan para este estudio cuatro experiencias de las carreras de Humanidades, Enfermería 
y Terapia Ocupacional, pues se considera que son las carreras con más trayectoria acumulada desde la puesta en marcha del ApS en UFS (Fourie, 2003). En resumen, estas experiencias y espacios explorados fueron los siguientes:

\section{ApS 1. Estudiantes de primer año de Enfermería (un grupo de 120 estudiantes)}

En poblado de Springfontein, ubicada $150 \mathrm{~km}$ sur del Campus Bloemfontein. Se determinan las necesidades sociales sentidas y generan alternativas de atención y prevención de las enfermedades, capacitación en saneamiento y terapias domésticas, alimentación saludable y medicación general, entre otros. Estudian aspectos éticos y legales de la profesión y del trabajo social. En varias sesiones in situ, realizan actividades de diagnóstico, investigación y diálogo reflexivo con las familias, enfocándose en los más vulnerables del hogar (infantes mayores de seis años y personas adultas mayores). Se compartió más profundamente el trabajo de dos estudiantes que atendieron una familia de etnia negra, de bajos recursos, integrada por una adulta mayor, su hija y el nieto de 6 años.

\section{ApS 2 y 3. Estudiantes de tercer año de Enfermería y Terapia ocupacional (con 5 estudiantes)}

Actividades formativas con la Fundación ROC (Reach our Community Foundation, www. rocfoundation.co.za) en diversos temas previamente acordados (como salud mental, higiene básica en el trabajo y el hogar), para incrementar las capacidades del personal y su grupo meta (padres de familia e infantes de bajos recursos, todo el grupo nativo con origen étnico negro).

\section{ApS 4. Estudiantes de primer año de humanidades (cinco estudiantes)}

Trabajos de sensibilización e información con centros educativos de primaria en zonas vulnerables, aledaños a Bloemfontein; participan escolares de etnia negra en un abordaje de problemáticas de drogadicción y bullying.

\section{Actividades de gestión académica (con 12 personas)}

Reuniones periódicas de planificación, programación, coordinación, reporte, rendición de cuentas y seguimiento de ApS, entre otros.

La tabulación de los datos se realizó de manera manual partiendo de lo establecido por el ECDH (Robeyns, 2017), el cual propone sustentar los juicios normativos a partir del análisis de las capacidades (libertades) y los funcionamientos (acciones) que logran las personas (el estudiantado). Por consiguiente, las categorías de análisis se centran inicialmente en los logros más valorados del estudiantado, asociados a las capacidades de Walker y McLean (2013). Se 
intenta visibilizar los seres y haceres/acciones que les resultan más valiosos (Sen, 2000), que dan cuenta de la transformación social que vive el estudiantado participante. En la categoría de factores de conversión y recursos influyentes en las capacidades estudiantiles, se identifican las potenciales alternativas de mejora institucional. La revisión documental permitió comprender los contextos de las experiencias, previas a la observación participante y validar los hallazgos encontrados en las acciones y los relatos de las personas. Las tres técnicas aplicadas permitieron la triangulación de la información.

\section{Análisis de resultados}

\section{Contribución a la expansión de las capacidades profesionales y personales}

A continuación, se presentan, en un primer apartado, los resultados que reporta el estudiantado asociado a las capacidades identificadas por Walker y McLean (2013). Para viabilizar la discusión se agruparon las ocho capacidades de la siguiente manera: i) Visión informada y a las habilidades y conocimientos, capacidades centradas principalmente en conocimientos valiosos para el ejercicio de la profesión con la debida orientación prácticas, comprensión de necesidades y lectura social crítica requerida; ii) Afiliación, resiliencia y lucha social colectiva, que se asocia al componente social, convicción, espíritu de lucha y compromiso de ayudar a otros; y finalmente, iii) Reflexividad emocional, integridad y seguridad y confianza, que reúne capacidades emocionales que favorecen la estabilidad personal para una actuación profesional integral, armoniosa y equilibrada.

Esta agrupación se realizó posterior a la tabulación de datos, debido a la detección de fuertes conexiones de los discursos entre algunas de las capacidades identificadas por Walker y McLean (2013), donde la agrupación permitía una mejor comprensión de los resultados. Tal observación de los datos tabulados permite, a las autoras, atreverse a afirmar la existencia de una inextricable relación dialéctica entre las capacidades que se agrupan, cuestión novedosa en los resultados de la presente investigación para continuar profundizando en las capacidades profesionales y personales, tanto en el plano teórico como metodológico. Posteriormente, se exponen algunos de los factores de conversión en los que la UFS debe centrar acciones a fin de ampliar y consolidar las oportunidades de desarrollo humano que permite el ApS en el estudiantado.

\section{Visión informada y las habilidades y conocimientos}

La visión informada es la capacidad más reconocida y valorada porel estudiantado entrevistado, pues consideran que los módulos del ApS le permiten avanzar. Más adelante se presentan algunos de los funcionamientos que dan cuenta de su avance en las capacidades de visión informada y habilidades y conocimientos. Las acciones de comunicación estrecha y horizontal entre las personas participantes (estudiantado, docentes y actores sociales), en torno a la realidad y las necesidades

12 
locales, mejora su visión informada y el fortalecimiento de habilidades de comunicación y de trabajo cooperativo. Mientras se comunican, capacitan, investigan, reflexionan y colaboran, mediados por el diálogo asertivo; el estudiantado hace una puesta en valor del sentido de su profesión; comprende la importancia de sus conocimientos técnicos y poder compartirlos adecuadamente con la comunidad. Con la mediación pedagógica, ApS les ayuda a lograr aprendizajes y dominio de herramientas que les facilitan realizar una lectura situada de la realidad social y tomar decisiones de manera sensible e informada. Hay un reconocimiento a la importancia de conseguir la conexión entre la teoría y la práctica y, por tanto, se valora positivamente la enseñanza que adquieren al combinar sus estudios en el aula con la realidad exterior.

Algunas evidencias de los funcionamientos estudiantiles logrados y asociados a visión informada y habilidades y conocimientos se reconocen en expresiones como:

- Aprendía combinar mis estudios con la realidad exterior; el proceso seguir para la redacción de propuestas [sociales], enseñándome la ética de cómo trabajar con una comunidad... a entender cómo es el trabajo con la realidad. (Estudiante 1)

- ...les demostramos los efectos del bullying, no solamente en ellos, sino en la víctima también... me di cuenta de que [los niños] necesitaban alguien con quien hablar en ese momento; fui capaz de trabajar con los miembros de mi grupo... trabajar con otras personas y después entender cómo se logra el trabajo cooperativo. (Estudiante 2)

- pude aplicar la visión dentro de un ejemplo práctico... tener una comprensión clara... mejorar como estudiante y conseguir que tengamos una comprensión clara de la teoría; Terminamos siendo capaces de superar cualquier obstáculo que tuvimos. (Estudiante 3)

El estudiantado desarrolla una serie de capacidades que contribuyen a una preparación académica más humanística, que eleva su potencial para ocupar roles sociales en el futuro. Hay logros satisfactorios en su protagonismo, en áreas de liderazgo, autonomía, determinación, toma de decisiones en equipo y búsqueda de medios para poner en acción su pensamiento crítico. En las entrevistas, sobresale la significancia del desarrollo de las habilidades de coordinación, organización y gestión de actividades comunales, experimentado mientras asumen roles y tareas en la comunidad. Esto fortalece algunos valores sociales que son importantes para una convivencia provechosa y armoniosa, por ejemplo, responsabilidad, solidaridad, tolerancia, justicia, servicio, respeto, cooperación, empatía, iniciativa, puntualidad, comunicación, planificación, entre otros. En los informes, se encuentran evidencias sobre cómo el estudiantado es capaz de utilizar medios creativos para comunicar sus mensajes, mediante el uso de juegos, canciones, bailes y dinámicas al aire libre, dependiendo de las posibilidades del grupo con que interactúa. También hay productos que demuestran el progreso en términos intelectuales, por ejemplo, producción de documentos publicables, tesis y ponencias en congresos (UFS, 2014). Al aumentarse los conocimientos y habilidades de investigación, trabajo en equipo, análisis de problemas sociales en contexto y comunicación dialógica, el estudiantado eleva su criticidad, 
confianza y determinación para desarrollar acciones sociales, por lo que también favorece una actuación más íntegra y responsable.

\section{Afiliación, resiliencia y lucha social colectiva}

El convivir en la comunidad con las personas en desventaja social, compartiendo con realidades adversas y complejas, ayuda a elevar sus niveles de sensibilidad hacia los demás, y estimula un encuentro sano de emociones y sentimientos que alimenta todas las capacidades identificadas por Walker y McLean (2013), entre ellas la de afiliación, resiliencia y lucha social colectiva. Esta experiencia promueve la generación de lazos afectivos, la ética de la profesión y el compromiso social. Tanto estudiantes como docentes expresan profundas preocupaciones por el reducido apoyo social en que viven las personas en pobreza; la soledad presente en los hogares, centros educativos y comunidades, y la falta de posibilidades reales para cambiar su condición de vida ${ }^{2}$. Hay expresiones de frustración y dolor por las escasas posibilidades individuales que poseen las personas de bajos recursos para resolver sus problemas sociales y la incapacidad que ha demostrado el Estado en la atención de las necesidades básicas de esa población; lo que sirve de evidencia del cuestionamiento sobre las estructuras de poder y cómo ejercen presión en los grupos con menores oportunidades económicas. Ante esto, el estudiantado asume un rol activo en la búsqueda de alternativas para mitigar los problemas locales y dar una respuesta oportuna.

Los vínculos afectivos y la confianza creados con las personas locales incrementan la cohesión, el compromiso y el esmero para que surjan desde el ApS contribuciones que sacien adecuadamente las expectativas de las comunidades. En las expresiones del estudiantado se destaca un interés genuino y aprehensivo por lograr superar barreras académicas, logísticas y de coordinación que permitan aportarles algo significativo a las comunidades. Esta disputa les lleva a vencer sus propios miedos, por ejemplo, a derivar las barreras del idioma. Hay que tener presente que, para comunicarse entre equipos, el estudiantado utiliza el idioma inglés y con las personas locales se necesitan otros idiomas (como Afrikaans, Ndebele, Sesotho y Zulú), lo que es una oportunidad de apropiación cultural y valoración de su identidad.

Los funcionamientos estudiantiles logrados asociados a afiliación, resiliencia y lucha social colectiva se relacionan con expresiones como:

- [ApS] me enseñó a ser mucho más responsable con mi trabajo ... a ser decisivo, a gritar si tengo que gritar ... a mejorar mi inglés, porque me daba miedo hablar delante de la gente; hemos jugado un papel muy importante para las vidas de esos niños. (Estudiante 1)

\footnotetext{
2 Por ejemplo, en el acceso a alimentos de buena calidad, a la salud, al trabajo, a la educación, a la vivienda digna $\mathrm{y}$ al entretenimiento.
} 
- He experimentado que, al trabajar con otras personas, hay que ser paciente con ellos...; entendícómo afecta [el bullying] a los niños, no sólo en la escuela sino a las familias también; [ApS] ayuda a saber cómo trabajar con otros, a construir entre nosotros; he aprendido que las comunidades realmente necesitan ayuda, necesitan orientación...Me habría gustado más enseñanzas para mejorar sus vidas. (Estudiante 2)

- Aprendía ir a la comunidad y mirar lo que necesitan y ayudarles a satisfacer esa necesidad; [la experiencia] hizo que nos sintiéramos muy bien acerca de nosotros mismos, me hizo sentir que sé; no era una especie de donación de cosas, se trata más de algo más significativo. No era caridad, sino que estaba ayudando a la comunidad misma para crecer y ayudar con las soluciones a sus problemas. (Estudiante 3 )

La capacidad de resiliencia es una de las capacidades que ha generado mayor dificultad de identificación en las expresiones del estudiantado; sin embargo, es posible encontrar su ligamen a otras capacidades como serían la lucha colectiva y la seguridad y confianza. El aumento de la responsabilidad, de la convicción y la determinación para ayudar a otros seres, de superar las barreras de idioma, de trabajar en equipo y el deseo de superación personal son parte de los ejemplos de resiliencia encontrados. Hay un interés que excede los objetivos académicos, reflejado en la necesidad de disponer de más tiempo para el trabajo en las comunidades y profundizar más en sus problemáticas locales. Variedad de estudiantes comentan sobre iniciativas que están gestando por otras vías (ONG, comunidad, familia, universidad) para dar respuesta a las necesidades sociales sentidas, lo que podría ser indicador de que con el ApS se está logrando un llamado efectivo a servir a la sociedad e incrementos en su capacidad de agencia.

Los módulos de ApS permiten comprender el daño que pueden causar el asistencialismo, la caridad y la generación de dependencias. Durante la reflexión en el aula, se enfatiza en lograr una relación honesta y sostenible con la comunidad. Se capacita al estudiantado para que no permita que las emociones (sus capacidades de afiliación y lucha social) les motiven a brindar soluciones de corto plazo que generen falsas expectativas a las familias y grupos con los que se trabaja. La universidad, al igual que la mayoría de las organizaciones en las que se desempeñarán a futuro, no posee recursos económicos para resolver todos los problemas locales, solo se dispone de conocimientos, servicios y experiencia para ayudar a las personas.

\section{Reflexividad emocional, integridad y seguridad y confianza}

Las evidencias denotan que la experiencia de ApS favorece que el estudiantado también logre aumentar su reflexividad emocional, integridad y seguridad y confianza. La experiencia les moviliza sufuerza interior transformadoray generativa, al enfrentarse conambientes desconocidos, no controlados, con situaciones complejas e inesperadas. Esta experiencia provoca una especie de ruptura, confrontación y cambio en su manera de pensar y de percibir a las personas pobres y su contexto. Muestran interés en mejorar su empatía y gestionar mejor los conocimientos, en 
comprender la realidad y lograr un mayor empoderamiento y participación en la solución de los problemas externos. También nacen aspiraciones de mejora personal y colectiva, y la necesidad de gastar las energías en acciones que generen soluciones realmente significativas para la gente. El estudiantado recalca su valoración positiva hacia la mejora que han logrado en sus actitudes y la sensibilización, convicción personal y confianza para vencer obstáculos que les restringen a la hora de conducir iniciativas beneficiosas para las comunidades. Durante el ApS, no solo aprenden de las condiciones adversas presentes en la comunidad, familia u organización con la que trabajan, sino que también de lo cotidiano, de las lecciones de vida que le da la comunidad a cambio de su trabajo, con su estilo de vida sencillo y poco consumista, con su trato amable, compasivo, generoso y lleno de afecto. La comunidad les enseña de valores para la vida, de espíritu de lucha, entusiasmo, agradecimiento, amistad y alegría, entre muchas otras cosas más.

En la narrativa del estudiantado se identifican algunos funcionamientos logrados, vinculados a la reflexividad emocional, integridad y seguridad y confianza, por ejemplo:

- este trabajo tocó realmente mis emociones ... He tenido que encontrarme conmigo mismo, mis fuerzas y mis debilidades. Uno piensa que está firme, pero cuando se enfrenta con la vida real se da cuenta que no, que uno no puede mantenerse; me está permitiendo poner en práctica lo que voy a hacer, está clarificándome lo que exactamente es que se necesita de mí si sigo con el trabajo social. (Estudiante 1)

- comprendí que yo quiero ayudar a la gente, me hizo comprender la razón por la que elegí esta facultad; me recordó por lo que vine aquí y el plan inicial era ayudar; me cambió mi perspectiva, mi percepción, mi forma de ver las cosas ... lo que hice me motivó y me cambió la vida. (Estudiante 2)

- he logrado algo este año que de hecho nunca he logrado en toda mi vida, he hecho una diferencia en la vida de otra persona o en parte. Ese es el mejor de los resultados; nos da más fuerza para manejar los problemas sociales fuera de nuestra institución [UFS]; estoy más dispuesta ahora a trabajar con los niños como trabajador social y ayudarles en lo que pueda. (Estudiante 3)

Para el estudiantado que no tiene buen dominio del idioma inglés, el ApS ha sido un respiro y una oportunidad de reconciliación personal. En lo cotidiano, el dominio del inglés divide al estudiantado, no obstante, cuando tienen que trabajar en el módulo ApS tienen que integrar a quienes hablan idiomas propios para lograr la comunicación con la sociedad. Esto no solo estimula la generación de vínculos de confianza y cohesión entre estudiantes, sino que favorece la aceptación étnica. Por otra parte, para quien sirve de intérprete, es un medio de reconciliación, pues sienten recibir más valoración y respeto por sus pares y encuentran motivos de orgullo de su origen, etnia, color de piel e identidad cultural. Esto sirve de ejemplo del potencial del ApS hacia una convivencia y ejercicio de la profesión más orientado a la justicia social, a una perspectiva más humanística y ética y, en consecuencia, a cultivar los valores democráticos (como equidad, justicia, derechos, libertades) y para vivir y servir desde una manera más justa y equilibrada.

16 Carmen Monge-Hernández, Alejandra Boni-Aristizábal y Merridy Wilson-Strydom

Los artículos de la Revista Electrónica Educare del Centro de Investigación y Docencia en Educación de la Universidad Nacional, Costa Rica, se comparten bajo términos de la Licencia Creative Commons: Reconocimiento, № Comercial, Sin Obra Derivada 3.0 Costa Rica. Las autorizaciones adicionales a las aquí delimitadas se pueden obtener en el correo: educare@una.cr 


\section{Algunos factores de conversión que influyen en el ApS}

La aplicación de los factores de conversión (Robeyns, 2017; Sen, 2000), con las técnicas desarrolladas, buscaba ofrecer recomendaciones a la institucionalidad ${ }^{3}$, adaptables a otras experiencias universitarias de interacción con la comunidad, como son las aproximaciones de extensión universitaria desarrolladas por las universidades latinoamericanas, desde 1918, con la Reforma de Córdoba. Por lo tanto, se pone énfasis en los factores de conversión de naturaleza socio institucional, que permiten integrar al sector universitario con las dinámicas comunitarias. En ese sentido, la UFS y el marco de la educación superior sudafricana determinan, en gran medida, la gama de oportunidades que se promueven en el estudiantado desde el ApS (UFS, 2006), propiciando las condiciones académicas y la dotación de recursos para su desarrollo. A partir de la iniciativa nacional de la educación superior de incorporar el community engagement, la UFS con la Política 06.1 y la acumulación de experiencia y capacitación de equipos, ha logrado establecer importantes fortalezas en esta área y ha alcanzado, con el ApS, una fusión real de las tres funciones, según la Figura 3.

Figura 3: Las tres funciones sustantivas de la educación superior y el ApS

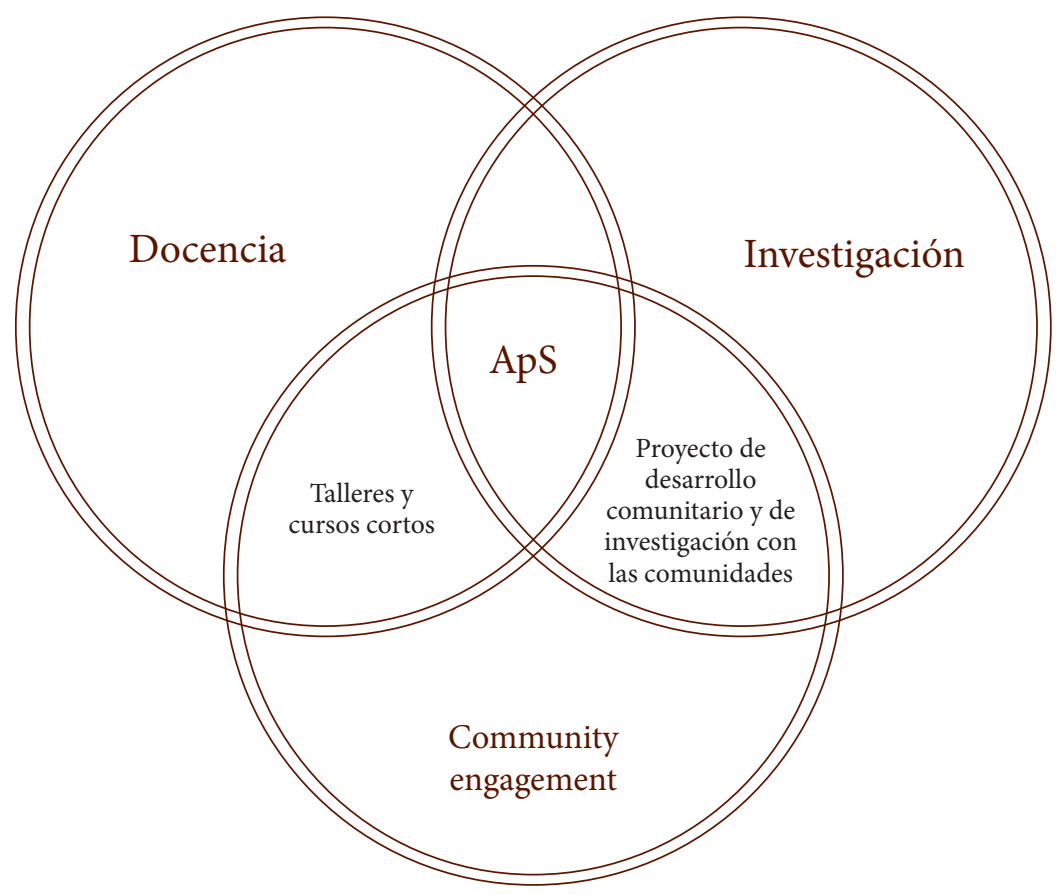

Nota: Elaboración propia a partir de UFS (2006).

\footnotetext{
${ }^{3}$ La cultura de la organización o institucionalidad incluye las normas sociales y las reglas formales e informales que rigen las acciones e interacciones sociales (Robeyns, 2017).
} 
Las entrevistas semiestructuradas aplicadas permitieron detectar algunos factores beneficiosos para la integración de los procesos sustantivos que genera el ApS, desde los criterios de las personas protagonistas. Al respecto, el ApS ha favorecido cambios sustantivos en el paradigma del conocimiento, al dar lugar a formas más amplias e inclusivas de generación de saberes desde la comunidad (Gestora 3). Esto ha permitido que el equipo vinculado al ApS comprenda, valore y proteja la promoción del intercambio promovido de los conocimientos científicos con las epistemologías y la cosmovisión local. Se reconoce el potencial del ApS para el aprendizaje y se avalan los logros alcanzados al participar y escuchar a los curanderos y curanderas (a quienes llama colegas locales) (Profesora 4). Se valora positivamente el trabajo conjunto logrado y las alianzas desde el saber local. En este sentido, hay convencimiento de que para lograr la transformación profesional y social que aspiran, en un país tan dividido como Sudáfrica, este trabajo es vital.

no se puede hablar de estas cosas solo en un aula y que toque el corazón del estudiante con tan solo hablar y decir cosas buenas de la transformación y el pensamiento transformacional. El estudiante tiene que salir y vivir y pasar por ese proceso transformador para lograr este sentido. (Gestora 3)

Se pone énfasis en la necesidad de utilizar nuevos enfoques para la interacción con la comunidad, de compromiso y respeto entre las partes; para la generación de vínculos y relaciones entre iguales, de doble vía o bidireccionales en los que tanto el equipo docente como el estudiantado y las comunidades tengan la libertad, la apertura y el interés para la enseñanza/ aprendizaje de unas a otras.

Si bien en los discursos de las personas participantes y en la normativa institucional se percibe consenso en la necesidad y la fortaleza del ApS, Ilama la atención que los equipos ponen en alerta los tropiezos que viven constantemente frente a restricciones estructurales vigentes. Hay indicios de que no hay interés en asegurar la financiación anual de los costos asociados al ApS (recurso humano, infraestructura, tecnología, transporte, equipos, materiales didácticos y logística). Hay, efectivamente, inestabilidad del financiamiento necesario a diferencia de la docencia y la investigación que mantienen la asignación de presupuestos debidamente planificados. Esto ha provocado incertidumbre, preocupación y desánimo; según la gestora 3 "todavía estamos implorando por ese apoyo, un poco más de apoyo de la dirección". Esto incentiva una cultura institucional que posterga y desvaloriza el community engagement como una función académica.

Lo anterior se reafirma en los dos últimos planes estratégicos donde el ApS está prácticamente ausente (UFS, 2012b; 2015). La voluntad política de apoyo es débil, según el representante de alto mando de la gestión académica, la UFS tiene objetivos más prioritarios 
que son de apremio para escalar en los estándares de competitividad mundial y excelencia académica. Si se toma en cuenta que cada año tienen que volver a insistir y convencer al equipo de gestión para que aprueben la financiación para el ApS, es comprensible encontrar, en los discursos, fuertes cuestionamientos acerca de si el ApS realmente constituye un componente estratégico para la UFS. La Gestora 1 reflexiona: "¿[el ApS]... sigue siendo estratégico o no? ... cada año hemos tenido la misma lucha de tratar de convencer [a los funcionarios de UFS] y a la gente nueva que viene, en que la financiación [al ApS] se debe dar de nuevo". Una parte del estudiantado y docentes, para hacer frente a los compromisos adquiridos con la comunidad, han optado por usar sus propios recursos, para comprar los materiales de trabajo, trasladarse en sus vehículos y recorrer largas distancia en carreteras con malas condiciones, en algunos casos con temor por su integridad física, en comunidades donde el ingreso es restringido ${ }^{4}$.

Otro aspecto que genera repercusiones negativas es la valoración académica y pedagógica; los méritos y el prestigio académico que recibe un profesor o una profesora por su trayectoria en ApS es desproporcionadamente menor respecto al concedido a quien se dedica a las funciones de docencia e investigación, lo que pone en desventaja al ApS. Según la Gestora 1, no hay un aprecio ni valoración real del trabajo con la comunidad, porque lo que la universidad busca son logros "más atrayentes, que hagan que la gente salte y en materia de participación comunitaria, no se supone algo impresionante o atrayente, es un trabajo humilde y humillante". En consecuencia, se necesita de un tipo de liderazgo académico particular, que interiorice y defienda el ApS. Esto, aunado a la adversidad en el factor financiero, alienta al sector docente a desplazarse y concentrarse en las otras funciones. Por ello, es posible decir que estos factores de la cultura institucional socavan el cumplimiento de los objetivos del ApS y la aspiración de avanzar hacia la integralidad (interdisciplariedad) y progresividad de los abordajes, que son parte de los encargos que le hace la comunidad a la UFS.

A modo de resumen, en la Figura 4 se presentan algunos de los factores de conversión descritos anteriormente.

Por otro lado, de acuerdo con las evidencias, los factores de conversión personales del estudiantado son muy heterogéneos y están determinados en gran medida por la cultura e historia de vida personal. Por el contexto, es transcendental el dominio de idiomas locales, la apertura mental y los valores sociales para hacer frente al desafío social; así como factores intra e interpersonales de las personas participantes que faciliten interactuar con otros seres con empatía, sensibilidad a los problemas ajenos, creatividad, confianza, autoestima, habilidades de socialización y comunicación, así como con respeto y horizontalidad dialógica. De manera positiva, esto se complementa con factores que aporta la comunidad, como las costumbres, normas sociales, aceptación y apertura, que además favorece el diálogo entre el saber popular y el científico.

\footnotetext{
${ }^{4}$ La estrategia para gestionar alianzas con el tercer sector y unir esfuerzos de la UFS con las demandas sociales vigentes aporta a la sostenibilidad de las acciones en comunidad, ante esa inestabilidad interna.
} 
http://doi.org/10.15359/ree.24-3.3

http://www.una.ac.cr/educare

educare@una.cr

Figura 4: UFS: Factores de conversión que favorecen o limitan la creación de capacidades mediante el ApS.

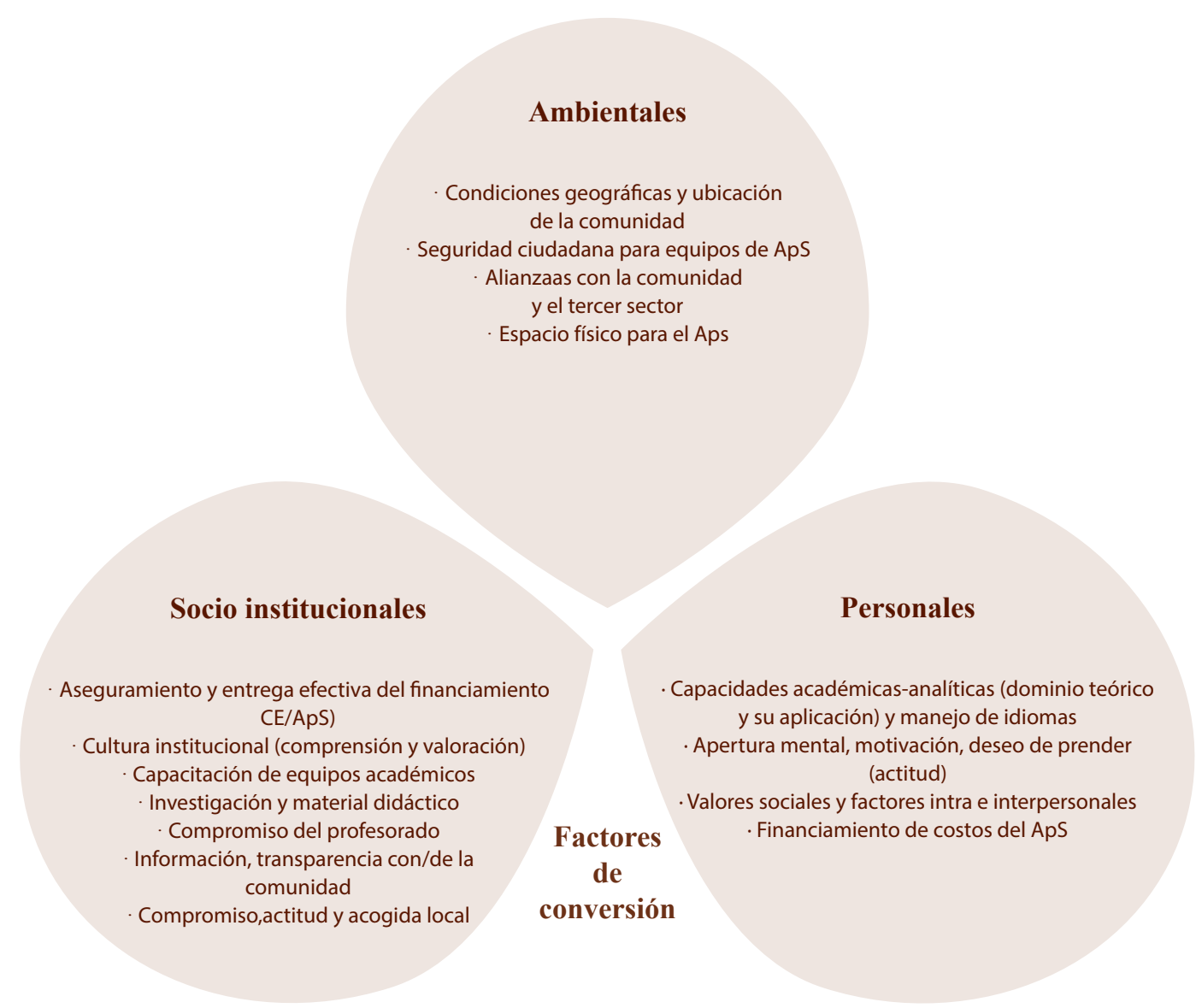

Nota: Elaboración propia.

Si bien el estudiantado y la comunidad participante presenta amplia pluralidad de factores de conversión, las evidencias apuntan a que es precisamente esa condición la que favorece ciertos aprendizajes positivos y avances en las capacidades humanas que se describen al inicio de este apartado de resultados.

\section{Discusión final}

La investigación muestra las aplicaciones del ECDH y las capacidades identificadas por Walker y McLean (2013), como recursos teóricos que han servido de base para el análisis empírico de los resultados del ApS en la UFS, en función de la ampliación de oportunidades para lograr el 
profesionalismo integral que demanda la sociedad actual. Las evidencias respaldan el potencial holístico y transformador del ApS, debido a la gran riqueza de aportación a las capacidades que juegan un papel significativo para el bienestar de la sociedad. Se demuestra compatibilidad entre el ApS y las orientaciones del ECDH, gracias a un proceso formativo que promueve el entendimiento de realidades dinámicas y complejas y el compromiso para la transformación social, más allá de concentrarse exclusivamente en la adquisición de competencias profesionales para satisfacer las demandas del mercado de trabajo.

Una vez concluido el análisis de la información, es posible señalar las implicaciones positivas del ApS en la ampliación de capacidades en áreas asociadas a las capacidades aportadas por Walker y McLean (2013), con énfasis en la adquisición y el fortalecimiento de valores base para una ciudadanía responsable, más conscientes de su compromiso con los desafíos sociales y aprehensivos de la función social de su profesión para la contribución al bienestar de otras personas. Consecuentemente y en concordancia con lo aportado por Sen (2000), es posible señalar que el ApS genera importantes contribuciones a los seres (valores, principios, anhelos, afecto, compasión y otras emociones positivas) y haceres/acciones (conocimientos, habilidades, destrezas y gestión de proyectos, entre otros) del estudiantado, todo lo cual es altamente beneficioso tanto a nivel individual como para la sociedad en general.

Si bien los resultados conseguidos por el estudiantado son positivos, es preciso señalar que esta investigación no se orienta a designar el ApS como el único medio en que las universidades pueden contribuir a formar grupos profesionales comprometidos con el desarrollo humano personal y las necesidades colectivas apremiantes. Mas aporta a (re)pensar la educación superior en función de integralidad y trasformación social que solicitan los problemas socioplanetarios vigentes.

La aplicación de los factores de conversión (Robeyns, 2017; Sen, 2000) permite identificar importantes deficiencias y limitaciones institucionales para garantizar la calidad y continuidad de las prácticas de ApS. Existe una contradicción solapada entre la normativa y la función de interacción con la comunidad en la UFS. Si bien es cierto que la UFS dispone de un marco legal institucional solvente, que permite que la universidad se integre a la dinámica local y genere beneficios en la formación humanística del estudiantado, se mantienen condiciones que restringen el aseguramiento de la estabilidad de estas prácticas y garanticen la ampliación de esas capacidades y logros profesionales socialmente favorables al futuro estudiantado egresado. Algunos de los potenciales cambios en la cultura institucional a promover apuntan al reconocimiento y a la valoración académica del community engagement como una función prioritaria de la academia, y lograr asegurar su financiación e incorporación de estas prácticas pedagógicas en el plan de estudios. Estas condiciones son consideradas por las personas entrevistadas como básicas para lograr que la UFS curricularice el ApS en todas las carreras, legitime y priorice estas prácticas académicas e incentive al funcionariado universitario de las demás carreras para que integren el ApS, y se consiga completar el ApS en la totalidad de los planes de estudios. 
Finalmente, otras recomendaciones apuntan a la necesidad de una mayor repercusión en la sostenibilidad de los procesos de desarrollo, la consecución de los fines de formación académica transformadora y el compromiso con la calidad de vida de las personas participantes del ApS. Para esto, el fortalecimiento del diálogo y las alianzas con agentes externos a la universidad conlleva implicaciones positivas, pues constituye un medio adecuado para fortalecer la participación, la generación de autonomía, la contribución universitaria en la atención de las prioridades de las comunidades, y la implementación de un abordaje más integral, planificado y progresivo de los problemas locales.

\section{Agradecimientos}

Este trabajo se dedica a todas las personas que aceptaron participar en las entrevistas y compartir sus experiencias de ApS con la UFS, en ONG, instituciones públicas, comunidades, aulas escolares y universitarias. Nuestro agradecimiento al personal de la Fundación ROC, al estudiantado, docentes y personal gestor universitario, en especial a la directora y equipo de investigación del Centre for Research on Higher Education and Development-UFS, por haber hecho posible este estudio de caso.

\section{Referencias}

Alarcón G. y Guirao, C. (2013). El enfoque de las capacidades y las competencias transversales en el EEES. Historia y comunicación social, 18, 145-157. https://doi.org/10.5209/rev HICS.2013. $\underline{\mathrm{v} 18.44318}$

Alkire, S. (2002). Dimensions of human development. World development, 30(2), 181-205. https:// doi.org/10.1016/S0305-750X(01)00109-7

Boni, A., y Gasper, D. (2011). La universidad como debiera ser. Propuestas desde el desarrollo humano para repensar la calidad de la universidad. Sistema. Revista de Ciencias Sociales, 220, 99-116. https://www.academia.edu/4971658/La Universidad como debiera ser. Propuestas desde el desarrollo humano para repensar la calidad de la Universidad

Corbetta, P. (2007). Metodología y técnicas de investigación social. McGraw-Hill.

Erasmus, M. A. (2007). Service learning: Preparing a new generation of scientists for aMode 2 society. Journal for New Generation Sciences, 5(2), 26-40. http://hdl.handle.net/10520/ AJA16844998 118

Erasmus, M. y Albertyn, R. M. (Eds.). (2014). Knowledge as enablement: Engagement between higher education and the third sector in South Africa. SUN Press. 
Fourie, M. (2003). Beyond the ivory tower: Service learning for sustainable community development: Perspectives on higher education. South African Journal of Higher Education, 17(1), 31-38. https://doi.org/10.4314/sajhe.v17i1.25189

Hall, M. (2010). Community engagement in South African higher education. En Community engagement in South African Higher Education (Serie Kagisano, n. ${ }^{\circ}$, pp. 1-52). Council on Higher Education. https://www.che.ac.za/sites/default/files/publications/Kagisano No 6 January2010.pdf

Lazarus, J., Erasmus, M., Hendricks, D., Nduna, J. y Slamat, J. (2008). Embedding community engagement in South African higher education. Education, Citizenship and Social Justice, 3(1), 57-83. https://doi.org/10.1177/1746197907086719

Monge, C. (2016). Análisis de las relaciones entre la universidad y la comunidad desde el enfoque de las capacidades para el desarrollo humano. El caso de la UFS [Tesina de Maestría]. Universitat Politècnica de València, España. http://digital.csic.es/bitstream/10261/162708/1/ UFSudafrica.pdf

Nussbaum, M. C. (2001). Women and human development: The capabilities approach. Cambridge University Press.

Preece, J., Gabo, P., Mmba, O y Osborne. M. (Eds.). (2012). Community engagement in African Universities: Perspectives, prospects and challenges. Leicester. NIACE.

Robeyns, I. (2017). Wellbeing, freedom and social justice: The capability approach re-examined. Open Book Publishers. https://doi.org/10.11647/OBP.0130

Santos, B. de S. (2007). La universidad en el siglo XXI. Para una reforma democrática y emancipadora de la universidad. Revista Umbrales, 15, 13-70. http://biblioteca.clacso.edu. ar/ar/libros/bolivia/cides/umbrales/15/umbrales15.html

Sen, A. (2000). Desarrollo y libertad. Editorial Planeta.

Suleman, F. (2016). Employability skills of higher education graduates: Little consensus on a much-discussed subject. Procedia-Social and Behavioral Sciences, 228, 169-174. https://doi. org/10.1016/j.sbspro.2016.07.025

UNESCO. (1998). Conferencia mundial sobre la educación superior: La educación superior en el siglo XXI: Visión y acción (Tomo 1. Informe final). Autor. http://unesdoc.unesco.org/ images/0011/001163/116345s.pdf

University of the Free State. (2006). Policy 06.1 Community Service Policy. https://www. ufs.ac.za/docs/default-source/all-documents/community-service-policy-107-eng. pdf?sfvrsn=e23e421 0 
University of the Free State. (2011). Service Learning Annual Report for 2011. Autor. https://www. ufs.ac.za/supportservices/departments/service-learning-at-our-university-home/servicelearning-information/annual-reports

University of the Free State. (2012a). Service Learning Annual Report for 2012. Autor. https:// www.ufs.ac.za/supportservices/departments/service-learning-at-our-university-home/ service-learning-information/annual-reports

University of the Free State. (2012b). Strategic plan 2012 - 2016. https://www.ufs.ac.za/docs/ default-source/all-documents/strategic-plan-931.pdf?sfvrsn=4

University of the Free State. (2013). Service Learning Office Annual Report for 2013. Autor. https:// www.ufs.ac.za/supportservices/departments/service-learning-at-our-university-home/ service-learning-information/annual-reports

University of the Free State. (2014). Community engagement annual report 2014. Autor. https://www.ufs.ac.za/docs/librariesprovider43/service-learning-documents/reportsdocuments/ce-annual-report-2014-28-may-final.pdf?sfvrsn=44cd21 2

University of the Free State. (2015). Strategic plan 2015-2020. Autor. https://www.ufs.ac.za/docs/ default-source/all-documents/ufs-strategic-plan-2015---2020.pdf?sfvrsn=0

Valles, M. S. (1999). Técnicas cualitativas de investigación social. Síntesis.

Walker, M. (2012). A capital or capabilities education narrative in a world of staggering inequalities? International Journal of Educational Development, 32(3), 384-393. https://doi. org/10.1016/j.jijedudev.2011.09.003

Walker, M. y McLean, M. (2013). Professional education, capabilities and the public good: The role of universities in promoting human development. Routledge. https://doi. org/10.4324/9780203083895

Wilson-Strydom, M. y Walker, M. (2015). A capabilities-friendly conceptualization of flourishing in and through education. Journal of Moral Education, 44(3), 310-324. https://doi.org/10.1 $\underline{080 / 03057240.2015 .1043878}$ 